\title{
CONTRAST ENHANCEMENT TECHNIQUES USING HistogRAM EQUALIZATION METHODS ON COLOR IMAGES WiTH POOR LightNING
}

\author{
Dr. A. Sri Krishna ${ }^{1}$, G. Srinivasa Rao $^{2}$ and M. Sravya ${ }^{3}$ \\ Department of Information Technology, R.V.R \& J.C College of Engineering, \\ Guntur, Andhra Pradesh \\ atlurisrikrishna@yahoo. $\mathrm{com}^{1}$ \\ gsr.rvregmail. $\mathrm{com}^{2}$ murukutlasravya@gmail. $\mathrm{com}^{3}$
}

\begin{abstract}
Histogram equalization (HE) is a simple and widely used image contrast enhancement technique. The basic disadvantage of $H E$ is it changes the brightness of the image. In order to overcome this drawback, various HE methods have been proposed. These methods preserves the brightness on the output image but, does not have a natural look. In order to overcome this problem the, present paper uses Multi-HE methods, which decompose the image into several sub images, and classical HE method is applied to each sub image. The algorithm is applied on various images and has been analysed using both objective and subjective assessment.
\end{abstract}

\section{KEYWORDS}

Contrast enhancement, Histogram equalization, Multi Histogram Equalization, Multi-threshold selection.

\section{INTRODUCTION}

Image contrast enhancement [1] and [2] is a classical problem in image processing and computer vision. Image enhancement is considered as a preprocessing step in many areas like video/image processing applications [17] and [18] speech recognition, texture synthesis etc. Enhancement techniques mainly fall into two broad categories: spatial domain methods and frequency domain methods [15]. Spatial domain techniques are more popular than the frequency based methods, because they are based on direct manipulation of pixels in an image. Myriad spatial domain methods have been developed for visualizing the effect. Some of these methods uses simple linear or non-linear intensity level transformation functions, whereas others use complex analysis of different image features such as the edge and connected component information.

Contrast enhancement problem in digital images can be resolved using various methodologies, but Histogram Equalization (HE) [15] technique is the widely used one. Histogram Equalization method flattens the histogram and stretches the dynamic range of intensity values by using the cumulative density function. However, there are major draw backs in Histogram Equalization [10] especially when implemented to process digital images. Firstly, HE transforms the histogram of the input image into a uniform histogram by distributing the entire range of gray levels uniformly over the histogram of an image, with a mean value that is in the middle of gray level range. Accordingly, the mean brightness of the output image is always at the middle - or near to it in the case of discrete implementation. In the case of images with high and low mean brightness 
value, there is a significant change in the view of the enhanced image. Secondly, histogram equalization performs the enhancement based on the global content of the image. In other words, histogram equalization highlights the borders and edges between different objects, but may reduce the local details of these objects, and not adequate for local enhancement. Another consequence for this mergence is the production of over enhancement and saturation artifacts [16].

In order to overcome these drawbacks, variants of Histogram Equalization method are proposed. These include Brightness BI Histogram Equalization Method (BBHE) [1],[5] and [11], Dualistic sub image Histogram Equalization Method (DSIHE) [2] ,[6] and [14], Minimum Mean Brightness Error BI Histogram Equalization Method (MMBEBHE) [3] and [14]. BBHE Method divides the image in to two sub images based on the mean intensity value of the input image, and Histogram Equalization technique is applied for each sub image separately. Based on the Equal Area Property DSIHE method decomposes the image in to two sub images and Histogram Equalization technique is applied for both the sub images. MMBEBHE method also decomposes the image in to two sub images based on the intensity value that yields minimum Absolute Mean Brightness Error (AMBE) [3]. Recursive Mean Separate Histogram Equalization Method (RMSHE) [19] and [22] decomposes the input image recursively based on mean, up to a level $r$, hence $2^{r}$ sub images are generated. These sub images are equalized individually to obtain the desired result. Recursive Sub-Image Histogram Equalization (RSIHE) [9] and [22] is a generalization of DSIHE, so the median-based histogram segmentation is performed more than once. RSIHE uses the medians of sub histograms instead of the means of sub-histograms. SubRegion Histogram Equalization (CESRHE) [20] is a contrast enhancement method which separates the intensity range of the histogram into $\mathrm{k}$ sub-regions and redistributes the pixel intensities based on Sub-Intensity Range of output histogram. This method enhances the contrast while preserving mean brightness. The weighted average multi segment histogram equalization method [21] first smooths the global histogram and decomposes it into multiple segments via optimal thresholds, and then HE is applied to each segment independently. These methods outperform the conventional methods by producing enhanced image with details preserving ability.

The paper is organized as follows. Section 2 presents a detailed description about different Histogram Equalization Variants. Section 3 introduces two methodologies using Multi Histogram Decomposition. Section 4 includes results and discussions. Finally, conclusion is presented in Section 5.

\section{HiSTOGRAM EQUALIZATION VARIANTS}

In this section, we briefly describe conventional histogram equalization [10] and its variant methods. The CHE method was the base for all the other methods. The BBHE, DSIHE and MMBEBHE methods use Conventional Histogram Equalization which will be later described in this section. Notice that all the above CHE Variants decomposes the input image into sub images based on some criteria, and each sub image is equalized separately. BBHE uses mean intensity value, equal area property for DSIHE and MMBEBHE uses minimum AMBE as a criteria for division of sub images.

\subsection{Conventional Histogram Equalization Method}

This sub section describes the CHE [15] for color images in detail, since this method is the core of the present work. The goal of CHE method is to distribute uniformly the entire range of gray levels over the histogram of an image, increasing the image contrast. 
Let $\mathbf{X}=\{X(i, j, l)\}$ is an input image with $L$ discrete gray levels $\left\{X_{0}, X_{1}, \ldots, X_{L-1}\right\}$, where $X(i, j, l)$ is the intensity of the image at the $3 \mathrm{D}$ position $(i, j, l)$ and $X(i, j, l) €\left\{X_{0}, X_{1}, . ., X_{L-1}\right\}, \mathrm{H}(\mathbf{X})=\left\{n_{0}\right.$, $\left.n_{1}, \ldots, n_{k}, \ldots, n_{L-1}\right\}$ is the histogram of the image and $\mathbf{N}$ is the total number of pixels in an image. The probability density function (PDF) of the image is given in equation (1)

$$
\mathrm{P}(\mathrm{k})=\mathrm{n}_{\mathrm{k}} / \mathrm{N}
$$

where $n_{k}$ is the number of pixels whose gray level is $X_{k}$.

Cumulative distribution function (CDF) can be calculated using the Probability Density Function (PDF) as shown in equation (2)

$$
\mathrm{C}(\mathrm{k})=\sum_{j=2}^{k} \mathrm{p}(\mathrm{j})
$$

Histogram equalization T[i] can be calculated using CDF by using equation (3)

$$
\mathrm{T}[\mathrm{i}]=\{(\mathrm{G}-1) /(\mathrm{a} * \mathrm{~b})\} *^{\mathrm{c}}[\mathrm{i}]
$$

where $G$ is the number of gray level in an image and a, b is number of rows and number of columns in the image.

\subsection{Brightness Preserving Bi-Histogram Equalization}

BBHE [11] first divides the input histogram $\mathrm{H}(\mathbf{X})$ into two sub-histograms $\mathrm{h} 1(\mathbf{x})$ and $\mathrm{h} 2(\mathbf{x})$ by using the mean $\boldsymbol{x}_{\boldsymbol{m}}$, where $\mathrm{h} 1(\mathbf{x})$ is associated with the gray levels $\left\{x_{0}, x_{1}, . ., x_{m}\right\}$ and $\mathrm{h} 2(\mathbf{x})$ is associated with the gray levels $\left\{x_{m+1}, x_{m+2}, \ldots, x_{l-1}\right\}$. Then it performs conventional histogram equalization on $\mathrm{h} 1(\mathbf{x})$ and $\mathrm{h} 2(\mathbf{x})$ independently.

\subsection{Dualistic Sub Image Histogram Equalization}

The basic idea used in the DSIHE method is similar to by the BBHE method. Dividing the input image into two sub-images and equalizes the histograms of the sub-images separately. Instead of dividing the image based on its mean gray level, the DSIHE method divides the image based on equal area property [13] i.e., both the sub images contain the equal number of pixels, being one dark and one bright.

\subsection{Minimum Mean Brightness Error BI Histogram Equalization Method}

This method also decomposes the input image in to two sub images and Histogram Equalization method is applied for both the sub images. In MMBEBHE we find the threshold level $l t$ which decomposes the image $I$ into two sub-images $I[0, l t]$ and $I[l t+1, L-1]$, so that the minimum brightness difference is achieved between the input image and the output image. The threshold level $l_{t}$ is the value that yields minimum AMBE. Once the input image is decomposed, both the sub images are enhanced separately using the Classical HE process

\section{Multi Histogram Equalization Methods}

Multi Histogram Equalization [7] and [8] is a technique in which the input image is divided in to multiple sub images and then Histogram Equalization technique is applied for each sub image. The intensity value that divides the image in to sub images is the optimal threshold [12] set. The optimal threshold can be calculated using different methods but here we use two methods to 
calculate the threshold set. The first algorithm calculates the optimal threshold set based on the within class variance and divides the input image in to different sub images based on the threshold set which is given in 3.1. The second algorithm divides the input image in to several sub images based on the optimal threshold set generated using Otsu method as shown in 3.2. The image is divided up to a scale $r$, so that $2^{r}$ sub images are generated. Where $r$ can any predefined value.

The main idea behind the Multi Histogram Equalization Methods is to find an optimal threshold set $\mathrm{T}^{\mathrm{k}}=\left\{\mathrm{t}_{1}{ }^{\mathrm{k}}, \mathrm{t}_{2}{ }^{\mathrm{k}}, \ldots \ldots . . \mathrm{t}_{\mathrm{k}-1}{ }^{\mathrm{k}}\right\}$. The given input image is divided in to two sub images based on the mean value. The optimal threshold set is then calculated for each sub image and the original image is then divided in to $\mathrm{K}$ sub images $\mathrm{I}\left[1_{\mathrm{s}}{ }^{1}, 1_{\mathrm{e}}{ }_{\mathrm{e}}\right], \ldots \ldots, \mathrm{I}\left[\mathrm{l}_{\mathrm{s}}^{\mathrm{k}}, 1_{\mathrm{e}}{ }^{\mathrm{k}}\right]$, where $1_{\mathrm{s}}{ }^{1}$ referred to the minimum threshold value in the first sub image and $1_{\mathrm{e}}{ }^{1}$ referred to the maximum threshold in the first sub image. After dividing the image in to sub images apply Histogram Equalization Method for all the sub images and combine the equalized images to obtain the desired result.

\subsection{Minimum Variance Multi Histogram Equalization}

Step 1: Calculate the histogram of the image.

Step 2: Based on the mean Brightness value, divide the histogram in to two classes.

Step 3: Calculate the within class variance for both the classes. Within class variance is calculated as shown in equation (4)

$$
\operatorname{Disc}(\mathrm{i})=\left(\mathrm{i}-\mathrm{I}_{\mathrm{m}}\right)^{2 *} \mathrm{p}(\mathrm{i})
$$

Where $I_{m}$ is the average of all the intensity values within each class.

p(i) is the probability density function and

' $\mathrm{i}$ ' is the intensity value.

Step 4: Consider the intensity value that yeilds Minimum Within Class variance and divide each class in to different sub images.

Step 5: Apply Histogram Equalization technique for all the sub images.

Otsu method [4] is used to perform histogram shape-based image thresholding. The method assumes that the input image contains two classes of pixels or bi-modal histogram i.e., foreground and background, and then calculate the threshold separating these two classes. The extension of multi-level thresholding is referred to as the Multi Otsu method, in which multiple threshold values are identified to divide the image in to multiple sub images.

\subsection{Optimal Thresholding Multi Histogram Equalization}

Step 1: Calculate the histogram of the image.

Step 2: Based on the mean Brightness value, divide the histogram in to two classes.

Step 3: Calculate the optimal threshold set using otsu[4] method.

Step 4: Based on the optimal threshold set divide the image in to different sub images.

Step 5: Apply Histogram Equalization technique for all the sub images.

\section{RESUlts AND Discussions}

It is always desirable to have subjective and objective assessment to compare contrast enhancement techniques. The subjective assessment is an assessment of quality where there is no pre established measure or standard and is based solely on the opinion of the evaluator. The 
quantitative measures are used for objective assessment. However we have used the Absolute Mean Brightness Error (AMBE) and Mean Squared Error (MSE) as the quantitative measures. The Absolute Mean Brightness Error is calculated as the absolute difference between the means of the input and output image. MSE is a statistical measure of randomness that can be used to characterize the texture of the input image.

\subsection{Subjective Assessment on Color Images}

Fig1 shows the original image and their corresponding contrast enhancement versions. The various HE methods are compared. Fig1(a) shows the jar image which is poor lightened. Generally HE method utilizes the dynamic range of the pixel values for maximum contrast. The main drawback of HE method is over illumination which is observed in Fig1(b). By applying BBHE method, the patterns in the jar are clearly visible than in HE method and is shown in Fig1(c).

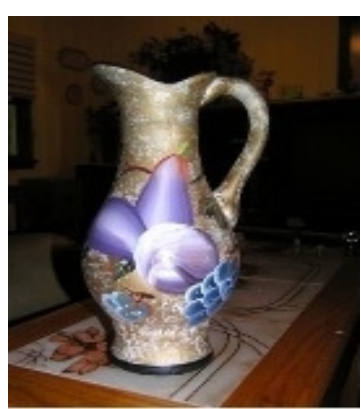

(a)

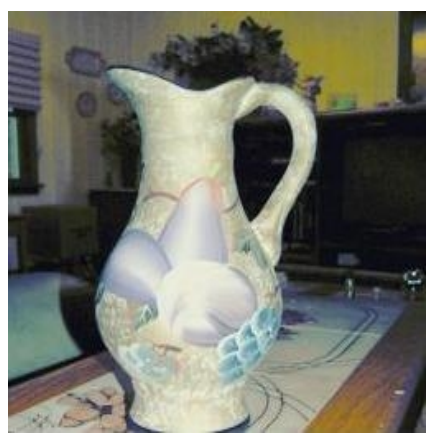

(e)

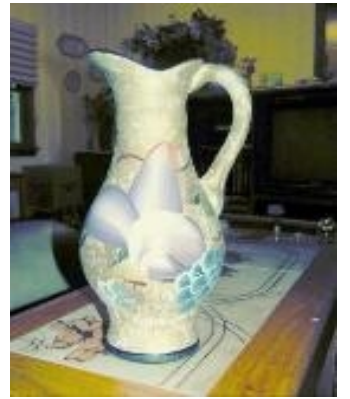

(b)

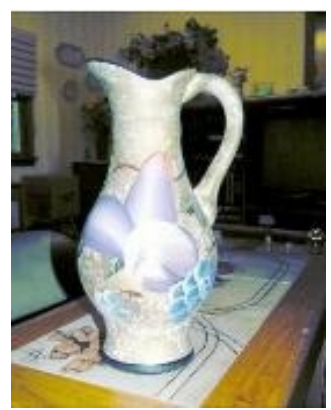

(c)

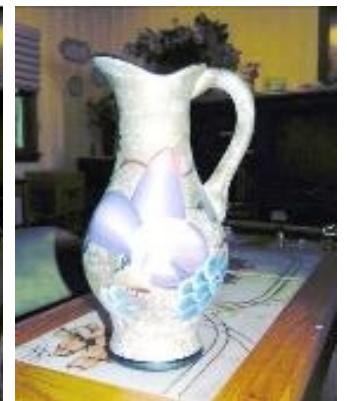

(d)

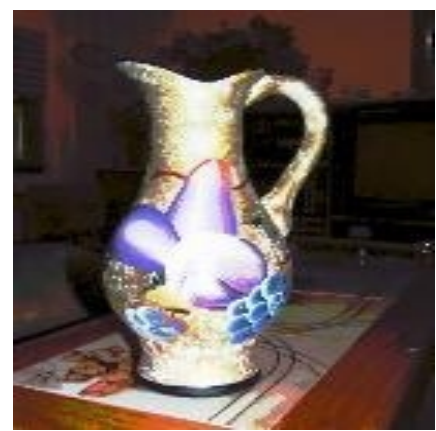

(f)

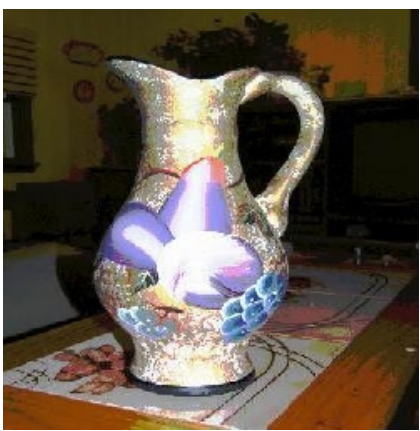

(g)

Fig1: Results for jar: (a) Original Image (b) Enhanced Image by applying HE Method (c) Enhanced Image by applying BBHE Method (d) Enhanced Image by applying DSIHE Method (e) Enhanced Image by applying MMBEBHE Method (f) Enhanced Image by applying MVMHE Method (g) Enhanced Image by applying OTMHE Method

In DSIHE Method, both the foreground and background parts are clearly visible than in the HE and BBHE methods. It can be seen in Fig1(d). In MMBEBHE method, the contrast is improved but the image does not have a natural look and it is shown in Fig 1(e). By applying the MVMHE method, the image has a natural look with a good contrast but there are unnecessary artifacts in the image. It is seen in Fig1(f). The OTMHE method preserves the brightness and increases the contrast of the entire image when compared to other methods and is shown in Fig1 (g). 


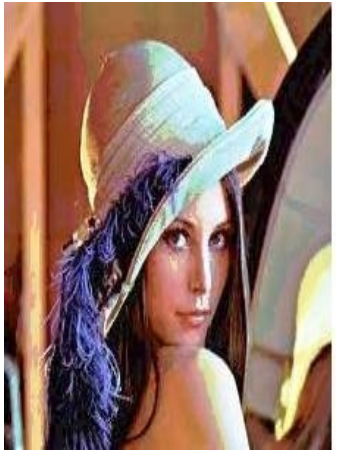

(a)

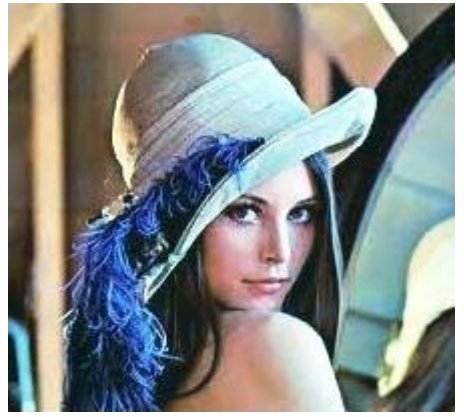

(e)

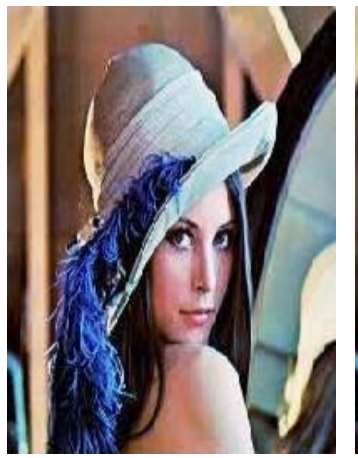

(b)

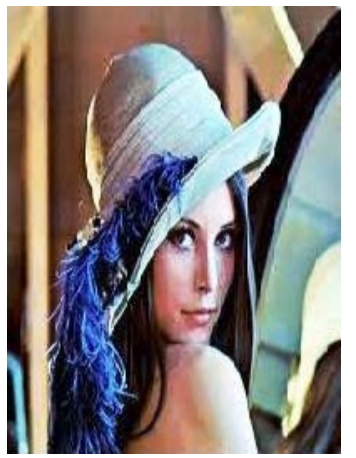

(c)

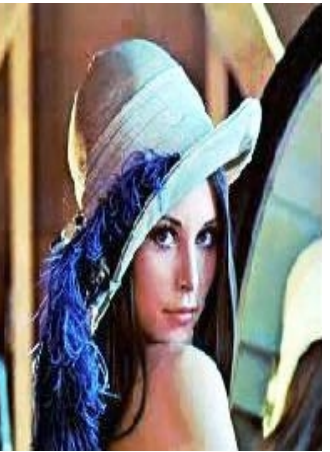

(d)

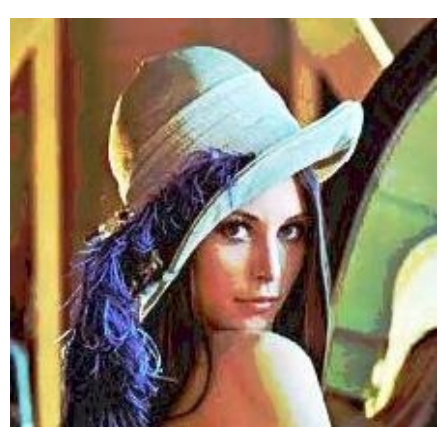

(f)

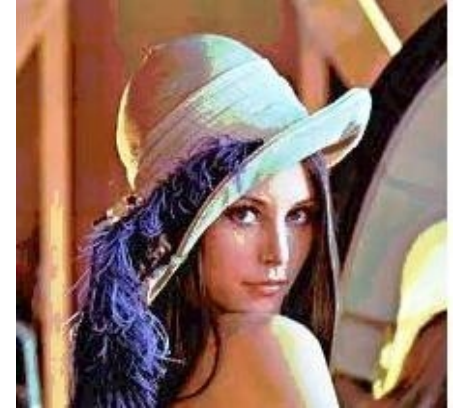

(g)

Fig 2: Results for Lena: (a) Original Image (b) Enhanced Image by applying HE Method (c) Enhanced Image by applying BBHE Method (d) Enhanced Image by applying DSIHE Method (e) Enhanced Image by applying MMBEBHE Method (f) Enhanced Image by applying MVMHE Method (g) Enhanced Image by applying OTMHE Method

Fig. 2 shows the Lena image and their corresponding contrast enhancement versions. The various HE methods are compared. Fig2(a) shows the original brightened image. HE method increases the contrast which results in over illumination of the image. This situation is observed in Fig2(b). By applying BBHE method, the foreground part of the image is more clearly which is shown in the Fig2(c). In DSIHE Method, the fore ground as well as the back ground has a pleasing look and is shown in Fig2(d). In MMBEBHE method, the contrast is only improved and the image does not have a natural look and is shown in Fig2(e). By applying the MVMHE method, the fore ground has a clear look and the background part contains unnecessary patterns as shown in Fig2(f).The OTMHE method preserves the brightness and increases the contrast of the entire image. It is observed in Fig2(g).

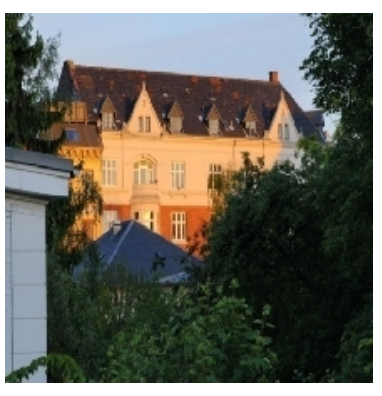

(a)

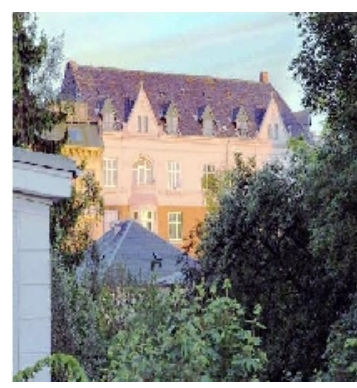

(b)

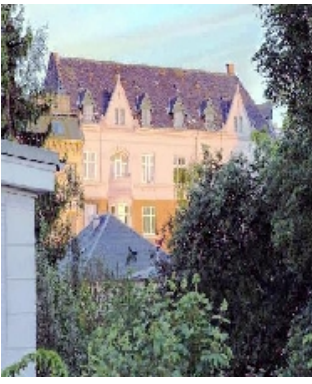

(c)

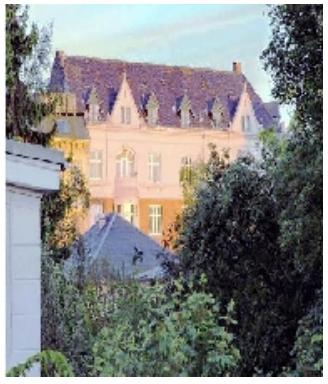

(d) 


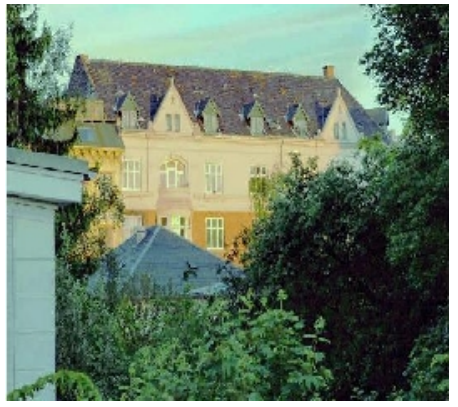

(e)

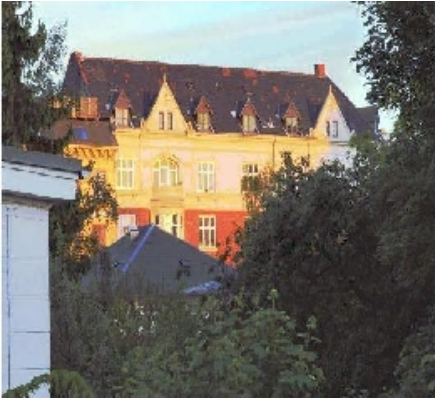

(f)

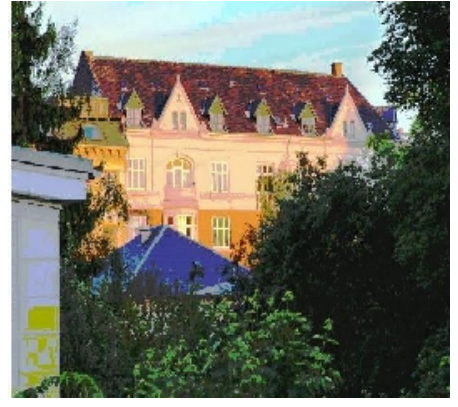

(g)

Fig3: Results for House: (a) Original Image (b) Enhanced Image by applying HE Method (c) Enhanced Image by applying BBHE Method (d) Enhanced Image by applying DSIHE Method (e) Enhanced Image by applying MMBEBHE Method. (f) Enhanced Image by applying MVMHE Method (g) Enhanced Image by applying OTMHE Method

Fig3 shows the House image and their corresponding contrast enhancement versions. The various HE methods are compared. Fig3(a) is the original image with good contrast. HE method enhances the image but the image does not have a natural appearance and shown in Fig3(b). By applying BBHE method, the foreground part is brightened as in Fig3(c). In DSIHE method, both the foreground and background are brightened more which can be observed in Fig3(d). In MMBEBHE method, the contrast is improved but brightness is not preserved. Hence the output image does not give a natural look which is shown in Fig3(e). By applying the MVMHE method, the contrast is enhanced and the background appears brighter as in Fig3(f).The MWCVMHE Method preserves the brightness and also improves the contrast of the image. It is observed in Fig3 $(\mathrm{g})$.

Fig4 shows the Sea image and their corresponding contrast enhancement versions. The various HE methods are compared. In Fig4 (a), there are very dark and bright regions. HE method enhances the darker regions but the brighter regions are over enhanced and do not have pleasing appearance. It is observed in Fig4 (b). By applying BBHE method, the brighter regions are over enhanced and the image does not have a natural look which can be observed in the Fig4 (c).In DSIHE method, the brighter regions are still over enhanced but the darker regions provides the details of the background part and is shown in Fig4 (d). In MMBEBHE method, the details in the darker region are hidden and only the brighter regions of the image are appeared as shown in Fig4 (e). By applying the MVMHE and OTMHE methods, the contrast is enhanced but there are some non existing artifacts in the images which does not gives the natural appearance. It is observed in Fig4 (f) and Fig4 (g).

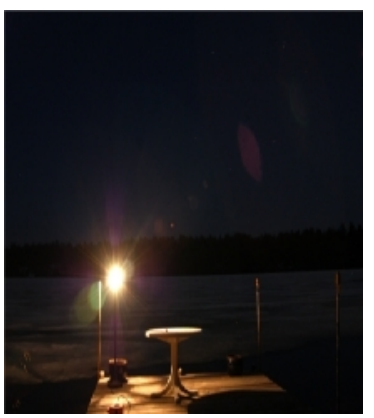

(a)

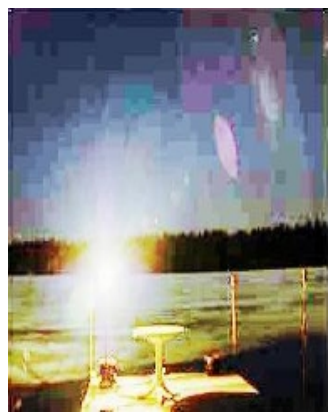

(b)

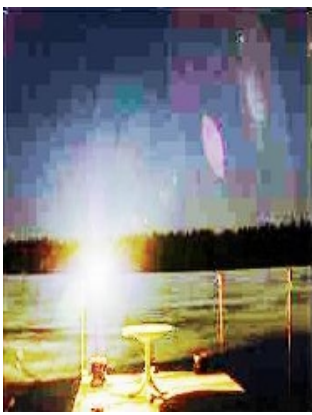

(c)

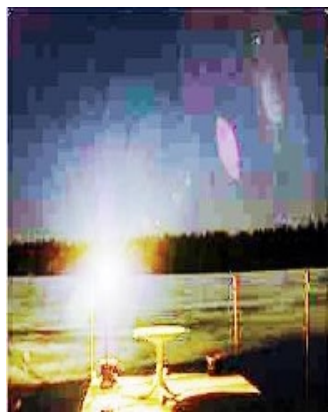

(d) 


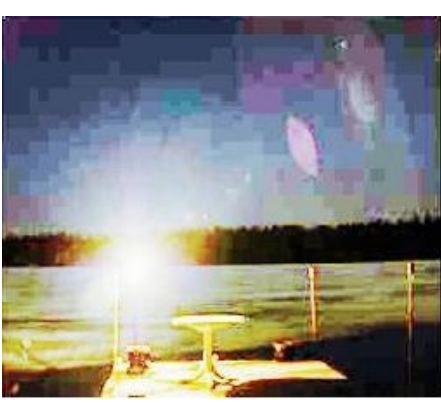

(e)

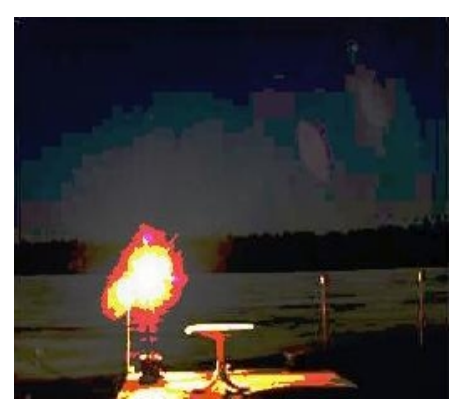

(f)

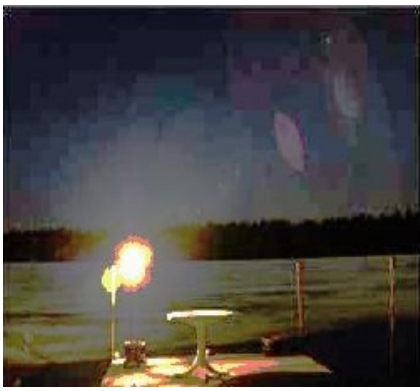

(g)

Fig 4: Results for Sea: (a) Original Image (b) Enhanced Image by applying HE Method (c) Enhanced Image by applying BBHE Method (d) Enhanced Image by applying DSIHE Method (e) Enhanced Image by applying MMBEBHE Method (f) Enhanced Image by applying MVMHE Method (g) Enhanced Image by applying OTMHE Method

\subsection{Objective Assessment on Color Images}

AMBE for all the intensity values with in an image is calculated.

$$
\mathrm{AMBE}=\left|\mathrm{M}_{\mathrm{i}}-\mathrm{M}_{\mathrm{o}}\right|
$$

Where, $\quad$ Mi is the mean intensity value of input image

Mo is the mean intensity value of output image.

Mean squared error (MSE) method is used to evaluate the performance of a predictor or an estimator. The mean squared error of the estimator or predictor is calculated as

$$
\operatorname{MSE}[\mathrm{T}(\mathrm{Y}) ; \mathrm{U}]=\mathrm{E}\left[(\mathrm{T}(\mathrm{Y})-\mathrm{U})^{2}\right]
$$

Where, $\mathrm{T}(\mathrm{Y})$ is the intensity value

$\mathrm{U}$ is the Mean intensity value of the input image.

\begin{tabular}{|c|c|c|c|c|c|c|}
\hline Method & HE & BBHE & DSIHE & MMBBHE & MVMHE & OTMHE \\
\hline Jug & 53.4377 & 49.92 & 64.1578 & 55.3254 & 19.9987 & 21.552 \\
\hline Lena & 9.6505 & 12.873 & 14.8883 & 10.5379 & 11.3436 & 10.1969 \\
\hline Sea & 100.6366 & 85.982 & 93.5739 & 73.982 & 11.1976 & 14.0923 \\
\hline House & 42.7876 & 38.928 & 41.9275 & 54.641 & 17.9376 & 29.0906 \\
\hline
\end{tabular}

Table 1: AMBE Values of Different Images 
International Journal of Computer Science, Engineering and Applications (IJCSEA) Vol.3, No.4, August 2013

\begin{tabular}{|c|c|c|c|c|c|c|}
\hline Method & HE & BBHE & DSIHE & MMBBHE & MVMHE & OTMHE \\
\hline Jug & 0.0089 & 0.0096 & 0.0130 & 0.0230 & 0.0013 & 0.0004 \\
\hline Lena & 0.0012 & 0.0011 & 0.0013 & 0.0041 & 0.0002 & 0.0003 \\
\hline House & 0.0015 & 0.0013 & 0.0014 & 0.0019 & 0.0011 & 0.0009 \\
\hline Sea & 0.0412 & 0.0315 & 0.0357 & 0.1398 & 0.0017 & 0.0011 \\
\hline
\end{tabular}

Table 2: Mean Square Error Values of Different Images

The various HE methods to enhance the contrast have been applied on different color images taken with different illumination. It has been observed that enhanced image using MVMHE Method gives good contrast but generates additional patches at the brightest region of an image. It has been observed that OTMHE Method results in generating natural look for the output image. The patches generated using MVMHE method has been taken care in the case of OTMHE method. The algorithm is basically used on the color images with poor lighting which helps us identifying those objects which are completely invisible in the images. It is obvious from Table 1 and Table 2 that Multi Histogram Equalization Methods produce minimum MSE and AMBE values when compared to all the other methods, which shows that these two methods are more efficient when compared to the other methods.

\section{CONCLuSion}

Multi Histogram Equalization technique improves image contrast by brightness preserving and generates natural looking images. An objective comparison among all the HE methods has been performed using quantitative measures AMBE (Absolute Mean Brightness Error) and Mean Square Error. It can be seen from the Tables that Multi Histogram Equalization Methods preserves the brightness more efficiently than other methods and the Mean Square Error is also reduced.

\section{REFERENCES}

[1] Y.-T. Kim, "Contrast enhancement using brightness preserving bi histogram equalization," IEEE Trans. on Consumer Electronics, vol. 43,no. 1, pp. 1-8, Feb. 1997

[2] Y. Wang, Q. Chen, and B. Zhang, "Image enhancement based on equal area dualistic sub-image histogram equalization method,” IEEE Trans. on Consumer Electronics, vol. 45, no. 1, pp. 68-75, Feb. 1999.

[3] S.-D. Chen and A. Ramli, "Minimum mean brightness error bi-histogram equalization in contrast enhancement," IEEE Trans. on Consumer Electronics, vol. 49, no. 4, pp. 1310-1319, Nov. 2003.

[4] N. Otsu, "A threshold selection method from grey-level histograms," IEEE Trans. on Systems, Man and Cyb., vol. 9 , no. 1, pp. 41-47, 1979.

[5] C. Wang and Z. Ye, "Brightness preserving histogram equalization with maximum entropy: A variational perspective," IEEE Trans. On Consumer Electronics, vol. 51, no. 4, pp. 1326-1334, Nov. 2005.

[6] C. Shannon, "A mathematical theory of communication,” Bell Syst. Tech. J., vol. 27, pp. 379-423, 1948.

[7] M. Luessi, M. Eichmann, G. Schuster, and A. Katsaggelos, "New results on efficient optimal multilevel image thresholding," in IEEE International Conference on Image Processing, 2006, pp. 773-776.

[8] David Menotti, Laurent Najman, Jacques Facon, and Arnaldo de A. Araújo "Multi-Histogram Equalization Methods for Contrast Enhancement and Brightness Preserving"

[9] Sim S., Tso P., and Tan Y., "Recursive Sub Image Histogram Equalization Applied to Gray Scale Images," Computer Journal of Pattern Recognition Letters, vol. 28, no. 10, pp. 1209-1221, 2007.

[10] Abdullah-Al-Wadud, M., Kabir, Md. Hasanul., Dewan, M. Ali Akber. and Chae, Oksam. (2007) "A Dynamic Histogram Equalization for Image Contrast Enhancement” IEEE 2007 
International Journal of Computer Science, Engineering and Applications (IJCSEA) Vol.3, No.4, August 2013

[11] Young-tack Kim and Yong-hun Cho, "Image Enhancing Method Using Men-Separate Histogram Equalization," United States Patent, Patent No. 5,963,665, Oct 5, 1999..

[12] J. S. Weszka, R. N. Nagel, and A. Rosenfeld, "A threshold selection technique."IEEE Trans.Comput., vol. C-23, pp. $1322-1326,1974$

[13] Yu Wan, Qian Chen and Bao-Min Zhang., "Image Enhancement Based On Equal Area Dualistic Sub-Image Histogram Equalization Method,” IEEE Trans Consumer Electronics, vol. 45, no. 1, pp. 68-75, Feb. 1999.

[14] Manpreet Kaur, Jasdeep Kaur, Jappreet Kaur "Survey of Contrast Enhancement Techniques based on Histogram Equalization" (IJACSA) International Journal of Advanced Computer Science and Applications, Vol. 2, No. 7, 2011

[15] R. Gonzalez and R. Woods, Digital Image Processing, 2nd ed. Prentice Hall, Jan. 2002.

[16] Iyad Jafar , and Hao Ying," Multilevel Component-Based Histogram Equalization for Enhancing the Quality of Grayscale Images", IEEE EIT, pp. 563-568, 2007.

[17] Pei C., Zeng C., and Chang H., "Virtual Restoration of Ancient Chinese Paintings Using Color Contrast Enhancement and Lacuna Texture Synthesis," Computer Journal of IEEE Transactions Image Processing, vol. 13, no. 3, pp. 416-429, 2004.

[18] Pizer M., "The Medical Image Display and Analysis Group at the University of North Carolina: Reminiscences and Philosophy," Computer Journal of IEEE Transactions on Medical Image, vol. 22, no.1, pp. 2-10, 2003.

[19] S.-D. Chen and A. Ramli, "Contrast enhancement using recursive mean separate histogram equalization for scalable brightness preservation," IEEE Trans. on Consumer Electronics, vol. 49, no. 4, pp. 1301-1309,Nov. 2003.

[20] Vinay Kumar, Nilesh, Tejeshwari Sahu," Contrast Enhancement using Sub-Regions Histogram Equalization " IJECT Vol. 2, Issue 3, sept. 2011.

[21] Khan, M.F. ; Khan, E. ; Abbasi, Z.A,”.Weighted average multi segment histogram equalization for brightness preserving contrast enhancement” Signal Processing, Computing and Control (ISPCC), 2012 IEEE International Conference.

[22] M. Kim and M. G. Chung:" Recursively Separated and Weighted Histogram Equalization for Brightness Preservation and Contrast Enhancement" Consumer Electronics, IEEE Transactions on (Volume:54, Issue: 3 ) August 2008

\section{AUTHORS}

Dr.A. Srikrishna received the AMIE degree in Electronics \& Communication Engineering from Institution of Engineers; Kolkata in 1990, M.S degree in Software Systems from Birla Institute of Technology and Science, Pilani in 1994, M.Tech degree in Computer Science from Jawaharlal Nehru Technological University (JNTU) in 2003. She has worked as lecturer from (1991-95). She is working in RVR \&JC College of Engineering, Guntur from 13 years as Assistant Professor and Associate Professor. She is pursuing her Ph.D from Jawaharlal Nehru Technological University (JNTU) in Computer Science under the guidance of Dr.V.Vijaya Kumar. Her research interest includes Image Processing and Pattern Recognition. She is Associate member of IE (I) and member of CSI.

G.Srinivasa Rao graduated in B.E(CSE) from Marathwada university, India in the year 1989, received masters degree M.Tech (CSE) from JNTU Hyderabad in the year 2008, M.s degree in software systems from Birla Institute of Technology and Science, Pilani in 1996 and he is pursuing PhD from JNTUH, Hyderabad. He has 23 years of teaching Experience. currently he is working as Associate Professor in the department of Information Technology, RVR \& JC College of Engineering, Guntur. His research interest includes Image and Signal Processing, algorithms and web technologies. He is a member

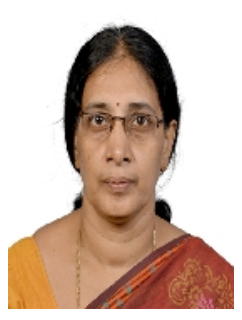
of CSI.

M.Sravya is Pursuing her B.Tech Graduation in Information Technology, in RVR \& JC College of Engineering, Chowdavaram, Guntur Andhra Pradesh, India. She has been actively presenting papers and participating in student technical Symposium seminars at National Level. Her area of interest includes Image Processing, Cloud Computing and Pattern Recognition.

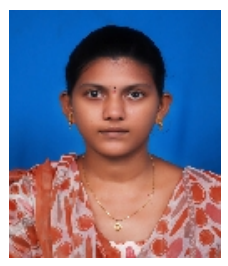

